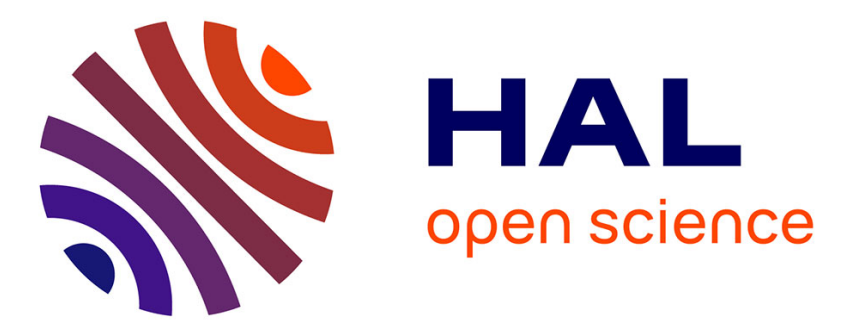

\title{
Les aberrations des miroirs bicylindres et leurs effets sur les propriétés des spectromètres de masse magnétiques à multiples passages \\ C. Berger, M. Baril
}

\section{- To cite this version:}

C. Berger, M. Baril. Les aberrations des miroirs bicylindres et leurs effets sur les propriétés des spectromètres de masse magnétiques à multiples passages. Revue de Physique Appliquée, 1979, 14 (8), pp.783-789. 10.1051/rphysap:01979001408078300 . jpa-00244660

\section{HAL Id: jpa-00244660 \\ https://hal.science/jpa-00244660}

Submitted on 1 Jan 1979

HAL is a multi-disciplinary open access archive for the deposit and dissemination of scientific research documents, whether they are published or not. The documents may come from teaching and research institutions in France or abroad, or from public or private research centers.
L'archive ouverte pluridisciplinaire HAL, est destinée au dépôt et à la diffusion de documents scientifiques de niveau recherche, publiés ou non, émanant des établissements d'enseignement et de recherche français ou étrangers, des laboratoires publics ou privés. 


\title{
Les aberrations des miroirs bicylindres et leurs effets sur les propriétés des spectromètres de masse magnétiques à multiples passages
}

\author{
C. Berger \\ Laboratoire de Physique Industrielle, I.N.S.A. de Lyon, 69621 Villeurbanne, Cedex, France
}

et M. Baril

Département de Physique et CRAM, Faculté des Sciences et de Génie, Université Laval, Québec, P. Qué., Canada G1K 7P4

(Reçu le 23 janvier 1979, révisé le 17 avril 1979, accepté le 7 mai 1979)

\begin{abstract}
Résumé. - Après un rappel des propriétés optiques paraxiales des miroirs bicylindres, de leurs applications aux spectromètres à multiples passages et de la définition des aberrations sphériques et chromatiques, on donne le résultat des calculs concernant les aberrations sphériques principales (i.e. lorsque l'objet est situé à l'infini) tant longitudinales que transversales. On présente ensuite le résultat pour un miroir dans les conditions effectives d'utilisation. Les aberrations chromatiques sont étudiées dans les mêmes conditions. L'accroissement progressif des aberrations avec le nombre de passages est ensuite présenté. En conclusion, il apparaît nécessaire d'apporter des modifications géométriques et électriques de manière à faire coïncider à la fois les conditions de transmission élevée et de faibles aberrations.
\end{abstract}

Abstract. - After recalling the paraxial properties of bicylinder mirrors and their application to multipassage magnetic mass spectrometers, results concerning spherical and chromatic aberrations are given. Both transversal and longitudinal spherical principal aberrations are presented. Results are shown for a mirror in actual operation. Chromatic aberrations have been studied in the same conditions. The increase of the aberrations with the number of passages is shown. In conclusion, it seems necessary to modify the mirrors both geometrically and electrically so that the conditions for good transmission and low aberration coincide.

1. Introduction. - Dans le cadre de l'étude du spectromètre de masse à multiples passages $[1,2]$ dont le principe est illustré par la figure 1 , nous avons

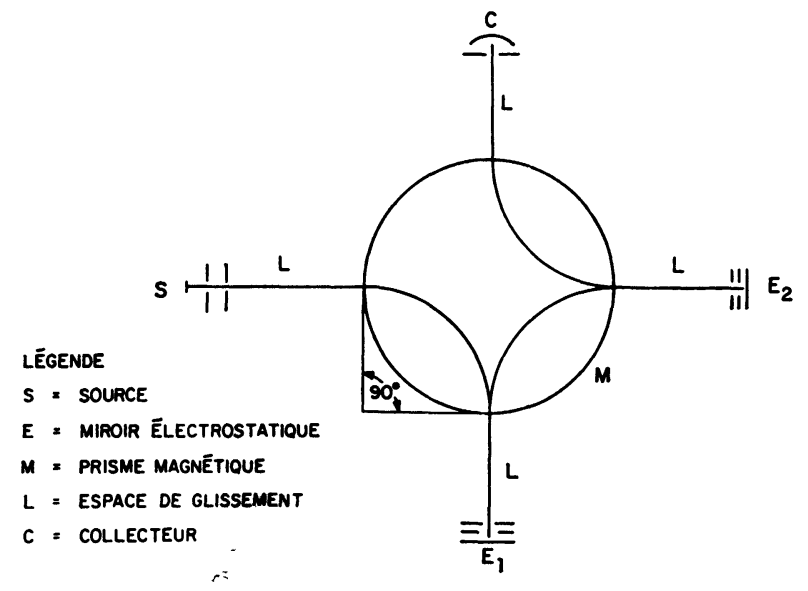

Fig. 1. - Schéma de principe du spectromètre de masse à multiples passages.

[Schematic diagram of multi-passage mass spectrometer.] dans la dernière publication [2] mis en évidence l'intérêt qu'il y aurait à utiliser des miroirs bicylindres (Fig. 2) pour l'obtention des réflexions nécessaires entre chaque passage dans le prisme magnétique.

L'étude était faite en approximation de Gauss et nous rappelons ici les deux aspects étudiés ainsi que les résultats fondamentaux déduits du calcul puis vérifiés par l'expérience.

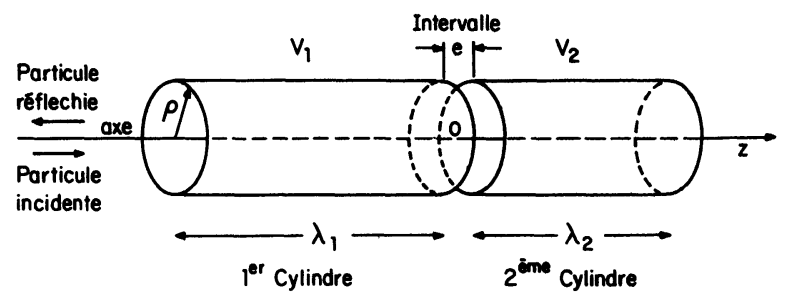

Fig. 2. - Miroir bicylindre. Les abscisses sont comptées à partir du point 0 centre de symétrie géométrique du miroir; le sens positif est le sens de déplacement des particules incidentes.

[Bicylindrical mirror. The coordinates originate at point 0 , the center of geometrical symmetry of the mirrors; the positive direction is that of the incident particle velocity.] 
Le premier point a été la détermination des caractéristiques optiques du miroir équivalent au miroir bicylindre en fonction du rapport $V_{2} / V_{1}$ des tensions appliquées aux deux cylindres composant le miroir. La figure 3 résume l'ensemble des résultats obtenus.

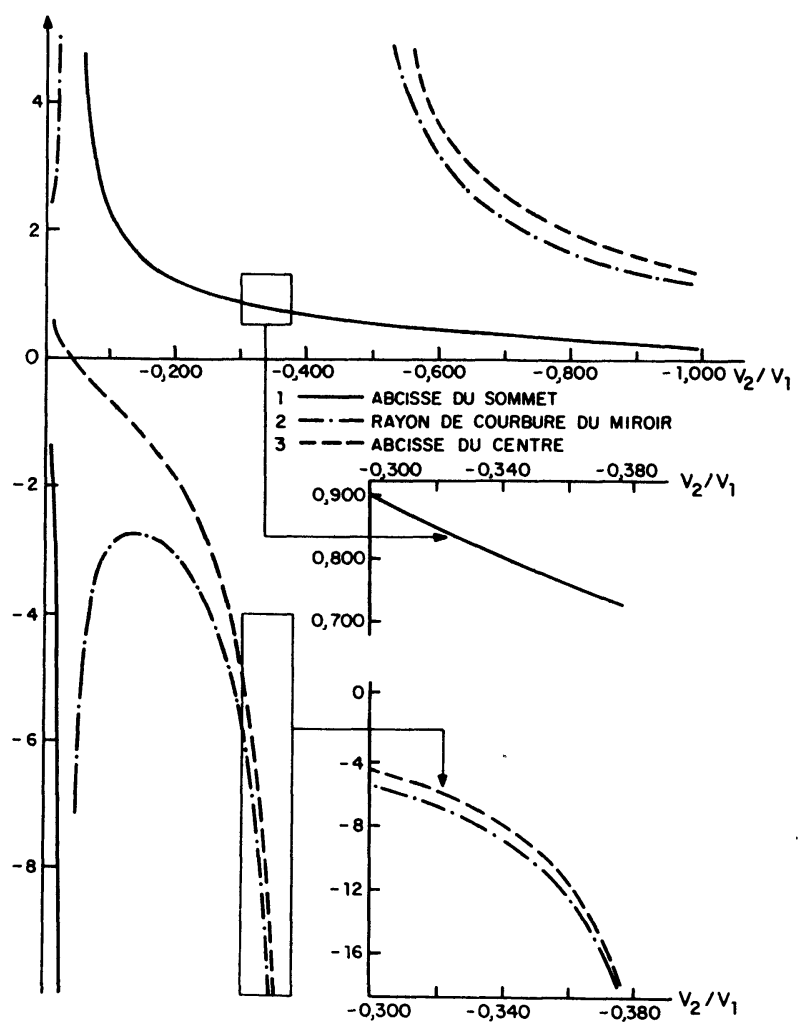

Fig. 3. - Caractéristiques optiques du miroir équivalent au miroir bicylindre en fonction du rapport $V_{2} / V_{1}$ des tensions appliquées aux cylindres.

[Optical characteristics of the mirror equivalent to the bicylindrical mirror with ratio $V_{2} / V_{1}$ between the voltages applied to the cylinders.]

Le deuxième point a porté sur l'examen du pouvoir de la transmission du spectromètre avec miroirs du type bicylindre. Les conclusions suivantes avaient été dégagées :

- la convergence nécessaire à ce système répétitif ne peut être obtenue que pour des rapports $V_{2} / V_{1}$ compris entre $-0,310$ et $-0,400$,

- à chaque valeur possible de $V_{2} / V_{1}$ correspond une géométrie optimale pour la transmission; le rayon du miroir équivalent doit alors être le double du rayon du prisme magnétique.

L'expérience avait également montré que les miroirs permettaient au moins trois passages, ce qui a été vérifié sur un montage avec déviation magnétique de $90^{\circ}$. Avec une telle valeur de la déviation, il n'est pas possible de réaliser plus de trois passages puisque au-delà le collecteur ou le quatrième miroir se superposerait à la source. Avec une déflexion légèrement plus faible, $80^{\circ}$ ou $85^{\circ}$ on pourrait envisager respec- tivement 8 ou 71 passages avant de trouver une position miroir ou collecteur confondue avec celle de la source.

Notre but est de réaliser un spectromètre avec le plus grand nombre possible de passages pour augmenter la résolution. Mais avant d'entreprendre un tel montage, il est nécessaire de connaître l'importance des aberrations et de leurs effets cumulatifs dont dépendra le nombre de passages utiles à une augmentation effective de la résolution.

Les aberrations des miroirs jouent un rôle perturbateur à la fois sur la luminosité du système liée essentiellement aux propriétés optiques du spectromètre dans le plan transversal, mais aussi sur la résolution qui dépend des propriétés optiques correspondant au plan radial. Dans ce plan s'ajoutent également les aberrations magnétiques.

Pour des raisons de simplicité des calculs, on étudie d'abord le comportement dans le plan transversal, où les seules aberrations sont celles des miroirs. Après cette étude partielle, nous verrons d'après l'ordre de grandeur des aberrations calculées s'il est utile de compléter les données sur les aberrations du spectromètre en étudiant le comportement dans le plan radial ou s'il est préférable d'envisager une autre solution.

L'étude est faite sur un système à déviation magnétique de $90^{\circ}$ en supposant le nombre de passages possibles supérieur à 3. Les indications que l'on en déduira seront valables pour les systèmes voisins.

Après un rappel des termes utilisés pour la définition des aberrations sphériques et chromatiques étudiées ici, nous analyserons d'abord les divers résultats concernant ces aberrations. Ces résultats sont obtenus par calcul des trajectoires selon le processus décrit dans la précédente publication. Ils ne concernent donc que les rayons se propageant dans la zone où l'on peut considérer le potentiel décrit par deux termes. C'est le cas des rayons transmis par le système, les plus externes étant éliminés au fur et à mesure des passages dans le prisme.

Enfin nous chiffrerons les effets cumulatifs au cours des passages successifs dans le prisme magnétique.

Les seules données sur les miroirs bicylindres sont à notre connaissance celles de Recknagel [3] malheureusement peu précises pour les raisons exposées dans [2]. Quant aux travaux de Kel'man, Fedulina et Yakuskev [4, 5] ils traitent de miroirs constitués non pas de cylindres mais de deux électrodes identiques juxtaposées et constituées chacune de deux plaques parallèles équidistantes.

\section{Rappel de définitions concernant les aberrations. -}

Les aberrations sphériques sont des aberrations d'ouverture correspondant à un point objet $A$ situé sur l'axe d'un système à symétrie de révolution.

Le point $A$ admet une image $A^{\prime}$ dont la position varie avec l'ouverture $\alpha$ du faisceau incident. $A_{\mathrm{p}}^{\prime}$ est l'image gaussienne (Fig. 4a). 


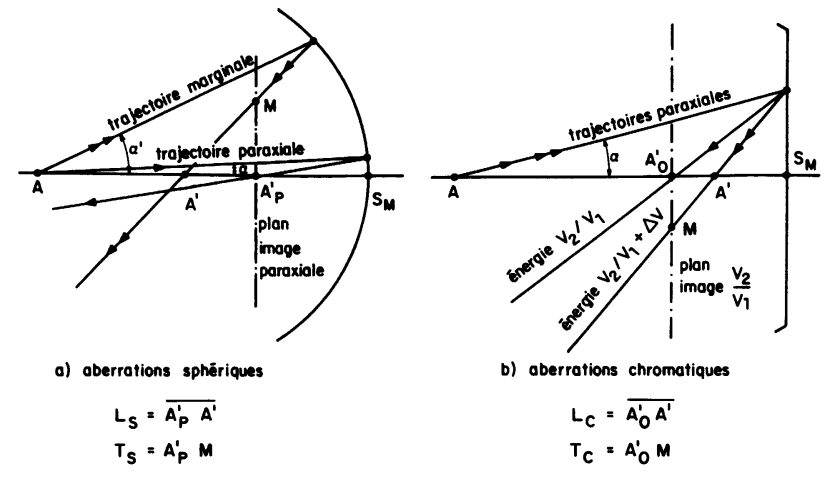

Fig. 4. - Définition des aberrations longitudinales $\left(L_{\mathrm{s}}, L_{\mathrm{c}}\right)$ et transversales $\left(T_{\mathrm{s}}, T_{\mathrm{c}}\right)$.

[Definition of longitudinal $\left(L_{\mathrm{s}}, L_{\mathrm{c}}\right)$ and transversal $\left(T_{\mathrm{s}}, T_{\mathrm{c}}\right)$ aberrations.]

Les deux grandeurs servant à définir l'importance de ces aberrations sont :

- l'aberration sphérique longitudinale

$$
L_{\mathrm{s}}=\overline{A_{\mathrm{p}}^{\prime} A^{\prime}} \quad \text { (grandeur algébrique) }
$$

- l'aberration sphérique transversale

$$
T_{\mathrm{s}}=A_{\mathrm{p}}^{\prime} M \quad \text { (grandeur positive) }
$$

cette dernière représentant la distance à l'axe de l'intersection $M$ du rayon marginal considéré avec le plan de l'image paraxiale.

$L_{\mathrm{s}}$ est une fonction paire de $\alpha^{\prime}$. En se rapportant à la figure 4 , on se rend compte que $T_{\mathrm{s}}$ est fonction de $L_{\mathrm{s}}$ et de $\alpha^{\prime}$. Il en résulte que $T_{\mathrm{s}}$ est une fonction impaire de $\alpha^{\prime}$, le premier terme étant en $\left(\alpha^{\prime}\right)^{3}$.

Lorsque le point objet est à l'infini, les aberrations qui s'y rapportent sont dites principales.

Les aberrations chromatiques décrivent les variations subies par l'image quand les particules incidentes ne sont pas monocinétiques. Ces variations d'énergie se traduisent par un changement de $V_{2} / V_{1}$. Il revient au même d'étudier le fonctionnement du miroir avec un rapport de tension $V_{2} / V_{1}$ subissant des variations désignées par $\Delta V$ autour d'une valeur centrale.

Un point objet $A$ sur l'axe a donc autant d'images gaussiennes $A^{\prime}$ qu'il y a d'énergies différentes pour les particules. $A_{0}^{\prime}$ est celle qui correspond au rapport fondamental $V_{2} / V_{1}$.

Pour définir ces aberrations (Fig. 4b), nous utilisons deux grandeurs semblables à celles définies précédemment :

- l'aberration chromatique longitudinale

$$
L_{\mathrm{c}}=\overline{A_{0}^{\prime} A^{\prime}} \quad \text { (grandeur algébrique) }
$$

- l'aberration chromatique transversale

$$
T_{\mathrm{c}}=A_{0}^{\prime} M \quad \text { (grandeur positive) } .
$$

La grandeur $T_{\mathrm{c}}$ est différente d'une donnée souvent rencontrée dans le cas des aberrations chromatiques, qui est le rayon du cercle de moindre diffusion.

$L_{\mathrm{c}}$ et $T_{\mathrm{c}}$ sont des fonctions de $\Delta V$, s'annulant pour $\Delta V=0$.

Remarque 1. - Les aberrations longitudinales sont comptées dans le sens de l'axe $\mathrm{O} z$ de la figure 2 . Une aberration longitudinale positive correspond à une augmentation de la convergence du miroir et une aberration négative à une diminution de la convergence.

Remarque 2. - Dans tout le texte, lorsque l'unité de longueur n'est pas spécifiquement précisée, il s'agit du rayon des cylindres constituant le miroir.

3. Aberrations sphériques principales. - Les aberrations sphériques longitudinales $L_{\mathrm{p}}$ et transversales $T_{\mathrm{p}}$ principales sont exprimées ici en fonction du rayon $h$ du faisceau cylindrique incident qui définit l'ouverture de ce dernier. Les développements limités s'écrivent

$$
\begin{aligned}
& L_{\mathrm{p}}(h)=a_{\mathrm{p}} h^{2}+b_{\mathrm{p}} h^{4}+\cdots \\
& T_{\mathrm{p}}(h)=c_{\mathrm{p}} h^{3}+\cdots
\end{aligned}
$$

$a_{\mathrm{p}}, b_{\mathrm{p}}, c_{\mathrm{p}}$ sont des coefficients indépendants de $h$, mais fonction des conditions de fonctionnement du miroir, c'est-à-dire du rapport $V_{2} / V_{1}$ des tensions appliquées aux deux cylindres. Les courbes $a, b, c$ de la figure 5 représentent les variations de ces trois coefficients en fonction de $V_{2} / V_{1}$.

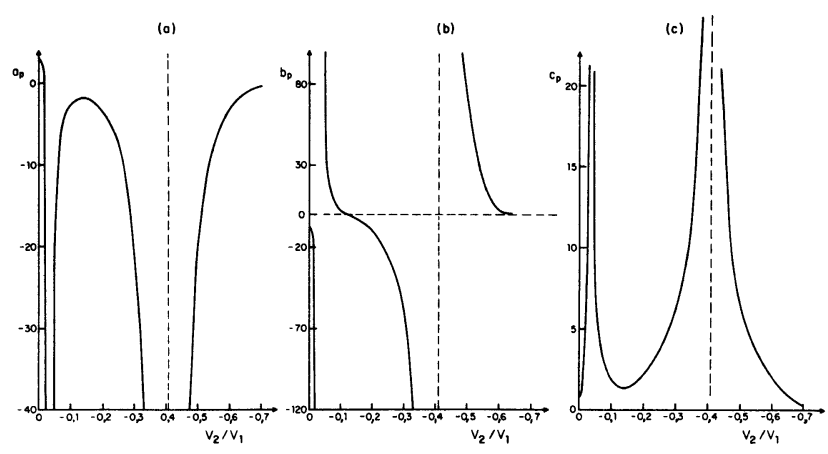

Fig. 5. - Courbes représentatives en fonction de $V_{2} / V_{1}$ des coefficients intervenant dans l'expression des aberrations sphériques principales $L_{\mathrm{p}}$ et $T_{\mathrm{p}}$.

[Typical curves of the coefficients appearing in the expression for the principal spherical aberrations $L_{\mathrm{p}}$ and $T_{\mathrm{p}}$ as a function of $V_{2} / V_{1}$.]

Le coefficient $a_{\mathrm{p}}$ est toujours négatif sauf peut-être pour des rapports $V_{2} / V_{1}$ inférieurs à $1 / 100$ en valeur absolue, le coefficient $b_{\mathrm{p}}$ change de signe suivant les valeurs de $V_{2} / V_{1}$ et le coefficient $c_{\mathrm{p}}$ est positif d'après la définition de $T_{\mathrm{p}}$.

Deux constatations s'imposent concernant ces variations :

- Les aberrations sont minimales dans les zones où le caractère (convergent ou divergent) du miroir 
est très affirmé. Cela se traduit par un minimum des coefficients $\left|a_{\mathrm{p}}\right|$ et $c_{\mathrm{p}}$ et une annulation du coefficient $b_{\mathrm{p}}$. Au contraire les valeurs de ces coefficients deviennent très grandes lorsque les miroirs ont un caractère peu affirmé.

- Les valeurs trouvées pour ces comportements particuliers des miroirs se produisent pour des valeurs de $V_{2} / V_{1}$ en plein accord avec les résultats concernant l'étude gaussienne des miroirs et représentés sur la figure 3 .

Dans cette étude, nous nous sommes limités à faire varier $h$ entre 0 et 0,25 . Le premier terme $a_{\mathrm{p}} h^{2}$ des aberrations longitudinales suffit à décrire celles-ci, lorsque $h$ ne dépasse pas une valeur $h_{\max }$ de 0,150 environ et lorsqu'il ne s'agit pas de région où la convergence du miroir en valeur absolue présente un extrémum. Dans les zones d'aberrations importantes, $h_{\max }$ devient de plus en plus faible au fur et à mesure que l'on s'approche des valeurs $V_{2} / V_{1}$ critiques. Au contraire $h_{\max }$ dépasse 0,150 lorsque le miroir est très convergent ou très divergent puisque $b_{\mathrm{p}}$ tend vers zéro.

4. Considérations sur le choix des caractéristiques du miroir et les conditions de fonctionnement de celui-ci. - D'après ce qui précède, pour réduire les aberrations sphériques principales au minimum, il faut faire fonctionner le miroir dans des conditions de convergence maximale, c'est-à-dire choisir un rapport $V_{2} / V_{1}$ le plus voisin possible de $-0,150$.

Malheureusement ce rapport de tension est incompatible avec le fonctionnement du spectromètre en système répétitif qui doit être convergent. La convergence ne peut en effet être obtenue que pour des rapports $V_{2} / V_{1}$ compris entre $-0,310$ et $-0,400$.

Enfin le dernier aspect dont il faut tenir compte est l'encombrement des miroirs placés autour du prisme magnétique, ce dernier ayant pour des raisons de

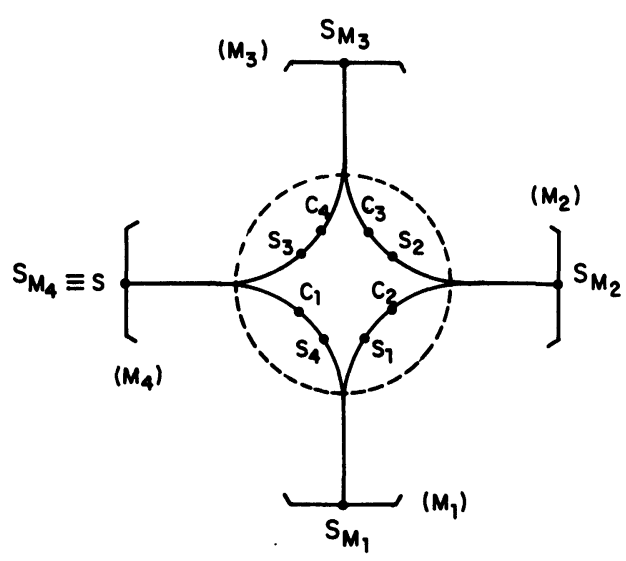

Fig. 6. - Position des éléments cardinaux des miroirs du spectromètre de masse à multiples passages, de la source $S$ et de ses images après les premières réflexions.

[Position of the cardinal elements of the multipassage mass spectrometer mirrors, of source $S$ and its images after the first reflections.] transmission optimale un rayon $R_{\mathrm{p}}$ moitié du rayon $R_{\mathrm{m}}$ du miroir équivalent.

Ceci nous a conduits à choisir le rapport du fonctionnement $V_{2} / V_{1}$ le plus proche possible de $-0,310$, qui soit compatible avec la réalisation pratique soit :

$$
\frac{V_{2}}{V_{1}}=-0,329 \text {. }
$$

Pour ce rapport, les caractéristiques du miroir convergent équivalent sont les suivantes :

abscisse du sommet $S_{\mathrm{M}}: \quad \overline{O S}_{\mathrm{M}}=+0,83$, abscisse du centre $C:$ : $\overline{O C}=-6,71$, abscisse du foyer $F \quad: \quad \overrightarrow{O F}=-2,94$, rayon du miroir $\quad: R_{\mathrm{M}}=7,54$.

Le second point à déterminer avant d'aborder le calcul des aberrations est la zone objet utilisée dans les miroirs au cours des divers passages.

Rappelons que dans le spectromètre à multiples passages la distance optique entre la source et le sommet du premier miroir équivalent ou entre les sommets de deux miroirs équivalents successifs est égale à deux fois le rayon $R_{\mathrm{M}}$ du miroir sphérique équivalent.

On désigne par $C_{\mathrm{i}}$ les centres des miroirs équivalents $\left(M_{i}\right)$ et par $S_{\mathbf{M}_{i}}$ leurs sommets, $S_{\mathbf{M}_{4}}$ étant dans le plan de la source $S$.

En appliquant les formules des miroirs sphériques, il est facile de constater que de la source $S$ située telle que $\overline{C_{1} S}=-R_{\mathrm{M}}$ le miroir $\left(\mathrm{M}_{1}\right)$ donne une image gaussienne $S_{1}$ telle que $\overline{C_{2} S_{1}}=-\frac{R_{\mathrm{M}}}{3}$. Le miroir $\left(\mathrm{M}_{2}\right)$ fournit à son tour de $S_{1}$ une image gaussienne $S_{2}$ telle que $\overline{C_{3} S_{2}}=-\frac{R_{\mathrm{M}}}{5}$ et ainsi de suite.

Les images gaussiennes de la source, obtenues par les réflexions successives, ont vis-à-vis des centres $C_{2}$, $C_{3}, C_{4}$ et $C_{1}$ des miroirs des abscisses

$$
R_{\mathrm{M}}, \quad-\frac{R_{\mathrm{M}}}{3},-\frac{R_{\mathrm{M}}}{5},-\frac{R_{\mathrm{M}}}{7}, \ldots
$$

(le sens positif étant le sens d'évolution des ions).

Elles convergent vers ces centres au fur et à mesure des passages dans le spectromètre.

La zone objet utile est donc comprise entre la source $S$ et le centre, ce qui correspond à $z$ évoluant entre $-14,1$ et $-6,7$. En fait nous étudierons ce qui se passe au delà du centre en direction du foyer jusqu'à $z=-4$ afin de pouvoir tenir compte des effets cumulatifs des aberrations.

5. Aberrations sphériques du miroir dans les conditions d'utilisation dans le spectromètre à multiples passages. - Les aberrations sphériques longitudinales $L_{\mathrm{s}}(\alpha)$ et transversales $T_{\mathrm{s}}(\alpha)$ peuvent s'exprimer sous 
forme d'un développement limité en fonction de l'ouverture angulaire $\alpha$ du faisceau incident

$$
\begin{aligned}
& L_{\mathrm{s}}(\alpha)=a_{\mathrm{s}} \alpha^{2}+b_{\mathrm{s}} \alpha^{4}+\cdots \\
& T_{\mathrm{s}}(\alpha)=c_{\mathrm{s}} \alpha^{3}+\cdots
\end{aligned}
$$

$a_{\mathrm{s}}, b_{\mathrm{s}}, c_{\mathrm{s}}$ étant des coefficients indépendants de $\alpha$, mais fonction de la position de l'image donc de celle de l'objet, repéré par rapport à 0 par son abscisse $z$.

Dans la zone étudiée $(-14,1 \leqslant z \leqslant-4,0)$ les coefficients $a_{\mathrm{s}}$ et $b_{\mathrm{s}}$ sont négatifs, le coefficient $c_{\mathrm{s}}$ est évidemment positif. Les courbes $a, b$ et $c$ de la figure 7 représentent les variations de ces trois coefficients en fonction de $z$.
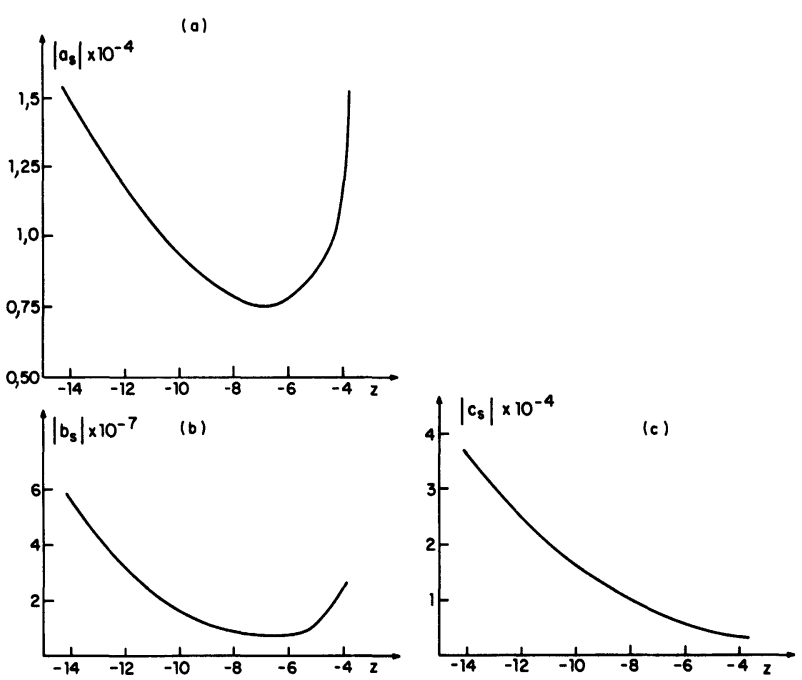

Fig. 7. - Représentation des coefficients servant à exprimer les aberrations sphériques $L_{\mathrm{s}}$ et $T_{\mathrm{s}}$ en fonction de l'abscisse $z$ de l'objet.

[Representation of the coefficients and to describe the spherical aberrations $L_{\mathrm{s}}$ and $T_{\mathrm{s}}$ as a function of the abscissa of the object.]

Les valeurs absolues de $a_{\mathrm{s}}$ et $b_{\mathrm{s}}$ décroissent depuis la position $z=-14,1$ jusqu'à $z=-6,7$ puis augmentent rapidement ensuite. Les aberrations longitudinales sont donc minimales lorsque l'objet est confondu avec le centre du miroir, elles deviennent très grandes lorsque l'objet tend vers le foyer objet où le moindre changement de conditions entraîne une grande variation dans la position de l'image.

Le coefficient $c_{\mathrm{s}}$ décroît constamment dans l'intervalle étudié, pour tendre vers une valeur minimale pour le foyer objet.

Il est à remarquer que malgré l'importance des coefficients, les aberrations sont d'un ordre de grandeur beaucoup plus faible, car ces coefficients sont associés aux facteurs $\alpha^{2}, \alpha^{4}$ ou $\alpha^{3}$ très petits devant l'unité. Ainsi au voisinage du centre pour un angle $\alpha$ de $5 \times 10^{-3} \mathrm{rd}$, l'aberration longitudinale $L_{\mathrm{s}}$ est de l'ordre de $-0,19$ et l'aberration transversale $T_{\mathrm{s}}$ de l'ordre de $0,9 \times 10^{-3}$.

On a également constaté que dans la zone étudiée les aberrations longitudinales se limitent au second ordre lorsque $\alpha$ ne dépasse pas 0,009 . Quant aux aberrations transversales elles se limitent à leur premier terme dans le domaine des ouvertures étudiées $(0 \leqslant \alpha \leqslant 0,025)$.

6. Aberrations chromatiques du miroir dans les conditions d'utilisation dans le spectromètre à multiples passages. - Les aberrations chromatiques longitudinales $L_{\mathrm{c}}$ et transversales $T_{\mathrm{c}}$ sont exprimées sous forme d'un développement limité en fonction de la variation $\Delta V$ du rapport de tension par rapport à la valeur de base $-0,329$

$$
\begin{aligned}
& L_{\mathrm{c}}(\Delta V)=a_{\mathrm{c}} \Delta V+b_{\mathrm{c}} \Delta V^{2}+\cdots \\
& T_{\mathrm{c}}(\Delta V)=c_{\mathrm{c}} \alpha \Delta V+d_{\mathrm{c}} \alpha \Delta V^{2}+\cdots .
\end{aligned}
$$

Dans cette formulation, les coefficients $a_{\mathrm{c}}, b_{\mathrm{c}}, c_{\mathrm{c}}, d_{\mathrm{c}}$, sont indépendantes de $\Delta V$ et de l'inclinaison $\alpha$ du rayon considéré. Ils sont fonction seulement de l'abscisse $z$ de l'objet.

Dans la zone étudiée $(-14,10 \leqslant z \leqslant-4,10)$ le coefficient $a_{\mathrm{c}}$ est positif et le coefficient $b_{\mathrm{c}}$ est négatif de sorte que les aberrations pour un même $|\Delta V|$ sont plus importantes dans le sens de l'accroissement de $\left|V_{2} / V_{1}\right|$ que dans le sens de la diminution de $\left|V_{2} / V_{1}\right|$. Cette observation est encore à relier au fait que quand $\left|V_{2} / V_{1}\right|$ diminue, le miroir a un caractère convergent plus affirmé donc moins sensible aux variations quelles qu'elles soient. Les coefficients $c_{\mathrm{c}}$ et $d_{\mathrm{c}}$ sont positifs.

Les courbes $a, b, c, d$ de la figure 8 nous renseignent sur les variations de ces coefficients en fonction de $z$. $a_{\mathrm{c}}$ et $\left|b_{\mathrm{c}}\right|$ sont croissants quand l'objet se déplace en direction du foyer et deviennent très grands à son approche. $c_{\mathrm{c}}$ et $d_{\mathrm{c}}$ sont décroissants jusqu'au centre $(z=-6,7)$ et augmentent rapidement ensuite.
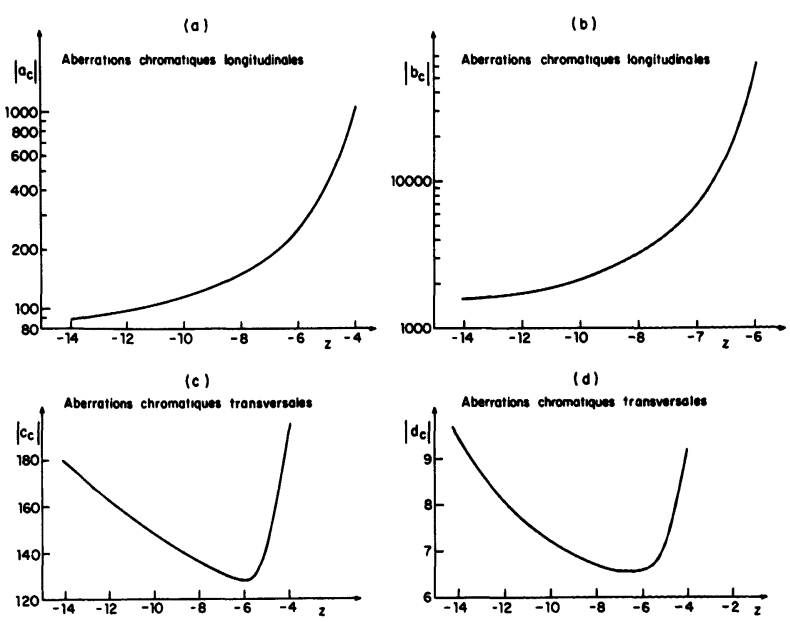

(d)

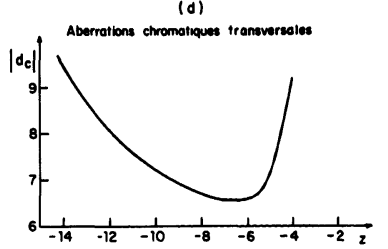

Fig. 8. - Représentation des coefficients intervenant dans l'expression des aberrations chromatiques $L_{\mathrm{c}}$ et $T_{\mathrm{c}}$ en fonction de l'abscisse $z$ de l'objet.

[Representation of the coefficients and to describe the chromatic aberrations $L_{\mathrm{c}}$ and $T_{\mathrm{c}}$ as a function of the abscissa of the object.] 
Dans l'étude, nous nous sommes limités à $|\Delta V| \leqslant 0,011$.

Deux termes $\left(\Delta V\right.$ et $\left.\Delta V^{2}\right)$ suffisent pour exprimer correctement l'aberration longitudinale $L_{\mathrm{c}}$ sauf lorsqu'elle devient très grande pour $z=-4$; les deux premiers termes ne sont alors valables que pour $|\Delta V| \leqslant 0,007$. Pour $T_{\mathrm{c}}$, deux termes suffisent toujours dans la zone étudiée.

7. Influence des aberrations dans le cas particulier d'utilisation des miroirs bicylindres. - Nous avons cherché à chiffrer l'importance des aberrations dans le cas particulier de l'utilisation des miroirs bicylindres pour le spectromètre de masse à multiples passages. On a donc suivi la marche de rayons subissant plusieurs réflexions.

Pour se rendre compte de l'influence des aberrations sphériques, il suffit de se reporter au tableau I, donnant les positions des images successives tout d'abord dans le cas idéal de rayons paraxiaux puis pour des angles d'incidence initiaux de $10^{-3}, 2 \times 10^{-3}$, $5 \times 10^{-3}$ et $10^{-2}$ rd. Dans le cas gaussien idéal, les images se rapprochent indéfiniment du centre, tout en gardant constamment une abscisse négative par rapport au centre. On voit que pour des inclinaisons même faibles, l'image se retrouve au contraire très vite au-delà du centre et il suffit alors de peu de réflexions supplémentaires pour que l'image se trouve alors au-delà du sommet suivant. Ceci modifie alors assez fondamentalement la forme du faisceau incident

Tableau I. - Abscisse des images successives du point source $S$ situé sur l'axe (origine : le centre, sens positif, le sens de propagation) en fonction de l'inclinaison initiale $\alpha$ des rayons formant ces images.

$\begin{array}{cccccc}\begin{array}{c}\text { Inclinaison } \\ -\end{array} & \overline{C_{2} S_{1}} & \overline{C_{3} S_{2}} & \overline{C_{4} S_{3}} & \overline{C_{1} S_{4}} & \overline{C_{2} S_{5}} \\ \text { Paraxiale } & -\overline{2,51} & -\overline{1,51} & -\overline{1,08} & -\overline{0,84} & -\overline{0,69} \\ 0,001 \mathrm{rd} & -2,50 & -1,43 & -0,79 & -0,38 & +0,08 \\ 0,002 \mathrm{rd} & -2,45 & -1,16 & -0,21 & +0,83 & \\ 0,005 \mathrm{rd} & -2,13 & -0,49 & +3,39 & & \\ 0,010 \mathrm{rd} & -0,40 & +2,77 & & & \end{array}$

qui habituellement divergent au niveau du miroir devient convergent. L'effet cumulatif des aberrations se fait d'autant plus rapidement sentir que l'inclinaison des rayons est plus importante au départ.

En ce qui concerne les aberrations chromatiques, deux comportements différents sont observés selon le signe de $\Delta V$, qui répétons-le, représente l'écart entre le rapport de tension de fonctionnement et le rapport origine $-0,329$.

Dans le cas où $\Delta V$ est négatif, ce qui correspond à des rapports de fonctionnement supérieurs en valeur absolue à 0,329 , le comportement est semblable à celui décrit pour les aberrations sphériques. Le tableau II permet de vérifier cette analogie et l'on peut là encore observer l'effet cumulatif très important des aberrations chromatiques.

Si l'écart $\Delta V$ est positif, les aberrations vont être de signe contraire. L'approche de l'image vis-à-vis du centre le plus voisin va donc être plus faible que pour le rapport $-0,329$. Les images successives ne convergeront plus au centre mais en un point d'abscisse négative par rapport au centre pour lequel l'avancement objet-image est exactement compensé par l'aberration. Ce point de convergence est d'autant plus éloigné du centre que $\Delta V$ est plus grand.

8. Conclusion. - De cette étude partielle sur les aberrations, on peut en tirer deux enseignements :

- Le premier a trait à la réalisation du spectromètre dont le principe est rappelé en début d'article.

Un tel spectromètre n'a d'intérêt que si le nombre de passages utiles est suffisamment important pour prétendre à une grande résolution. Les deux derniers paragraphes ont souligné l'ampleur des aberrations due à l'effet cumulatif de celles-ci. Il est donc indispensable de concevoir pour les miroirs des systèmes soigneusement corrigés de leurs aberrations.

- Le deuxième enseignement concerne l'emploi en général d'un système bicylindre comme miroir convergent.

Les variations brutales des caractéristiques avec le rapport $V_{2} / V_{1}$, l'exemple numérique traité ici montrent que les seules aberrations géométriques au niveau $\mathrm{du}$ point de rebroussement sont très importantes. Une analyse des origines des aberrations [7] montre

Tableau II. - Abscisse des images successives du point source $S$ situé sur l'axe en fonction de l'écart $\Delta V$ entre le rapport de tension de fonctionnement et la valeur - 0,329 prise comme origine.

\begin{tabular}{|c|c|c|c|c|c|c|c|}
\hline$\Delta V$ & $\overline{C_{2} S_{1}}$ & $\overline{C_{3} S_{2}}$ & $\overline{C_{4} S_{3}}$ & $\overline{C_{1} S_{4}}$ & $\overline{C_{2} S_{5}}$ & $\overline{C_{3} S_{6}}$ & $\overline{C_{4} S_{7}}$ \\
\hline - & - & - & - & - & - & - & - \\
\hline 0 & $-2,51$ & $-1,51$ & $-1,08$ & $-0,84$ & $-0,69$ & $-0,58$ & $-0,51$ \\
\hline$-0,001$ & $-2,42$ & $-1,32$ & $-0,83$ & $-0,49$ & $-0,27$ & $-0,05$ & $+0,18$ \\
\hline$-0,002$ & $-2,33$ & $-1,17$ & $-0,57$ & $-0,14$ & $+0,30$ & & \\
\hline$-0,005$ & $-2,03$ & $-0,59$ & $+0,35$ & & & & \\
\hline$-0,010$ & $-1,37$ & $+0,21$ & & & & & \\
\hline
\end{tabular}


que la distribution de potentiel de ce système présente toutes les caractéristiques d'un miroir à aberrations importantes. Il est donc préférable d'avoir recours à un système différent.

Compte tenu de ces conclusions quelque peu négatives vis-à-vis du but recherché, nos études se sont orientées vers l'élaboration de systèmes soigneusement corrigés des aberrations.

La première phase de ce travail a été le développement pour les systèmes cylindriques d'une technique de calcul du potentiel sur l'axe et de ses dérivées extrêmement rapide quelle que soit la complexité du système [6]. De plus cette méthode fait apparaître la contribution de chaque paramètre géométrique ou électrique du système.

Dans une deuxième phase, l'origine de chaque aberration a été analysée et reliée à la distribution de potentiel sur l'axe [7].

Enfin la troisième étape en cours d'achèvement est consacrée à la conception de systèmes où l'on a remédié dans une large mesure aux causes responsables des divers défauts d'un système (aberrations géométriques et chromatiques, mauvaise acceptance).

\section{Bibliographie}

[1] Fortin, M., Baril, M., Rev. Sci. Instrum. 43 (1972) 1140.

[2] Berger, C., Baril, M., Revue Phys. Appl. 13 (1978) 271.

[3] Recknagel, A., Z. Phys. 104 (1937) 381.

[4] Kel'man, V. M., Fedulina, L. V., Yakushev, E. M., Sov. Phys. Tech. Phys. 16 (1972) 1171, 16 (1972) 1449.
[5] Glikman, L. C., Kel'man, Y. M., Fedulina, L. V., YakuSHEv, E. M., Sov. Phys. Tech. Phys. 17 (1973) 1573.

[6] Berger, C., LAMBINET, M., BARIL, M., à paraître dans Revue Phys. Appl.

[7] Berger, C., Lambinet, M., Baril, M., en cours de rédaction. 